Original paper

\title{
Serum level of interleukin-13 receptor alpha 2 in infants with biliary atresia - is it of value?
}

\author{
Nermin Adawy ${ }^{1}$, Hanaa El-Araby', Alif Allam¹, Soha Elshenawy², Mohammed Khedr'1, Yasmine Ibrahim³, \\ Haidy M. Zakaria ${ }^{4}$ \\ 'Department of Pediatric Hepatology, Gastroentrology and Nutrition, National Liver Institute, Menoufia University, Egypt \\ 2Department of Biochemistry, National Liver Institute, Menoufia University, Egypt \\ ${ }^{3}$ Pediatric Department, Kfr Shokr Central Hospiral, Ministry of Health, Kaliobyia, Egypt \\ ${ }^{4}$ Pediatric Department, Quesna Central Hospital, Ministry of Health, Menoufia, Egypt
}

\begin{abstract}
Aim of the study: We aimed to assess the utility of serum level IL-13R 2 receptors as a non-invasive marker for early diagnosis of biliary atresia (BA) and selection of BA patients indicated for Kasai portoenterostomy.

Material and methods: The study included 60 infants with neonatal cholestasis in three groups; early BA group $(n=20)$, delayed BA group $(n=20)$ and non-BA cholestasis group $(n=20)$. A fourth group of 20 healthy neonates $(n=20)$ served as controls. IL-13Ra2 was measured by enzyme-linked immunosorbent assay in all patients and controls.

Results: The mean value of IL-13R $\alpha 2$ was significantly higher in delayed BA group (11.05 $\pm 10.9 \mathrm{ng} / \mathrm{ml})$ compared to early BA $(0.34 \pm 0.37 \mathrm{ng} / \mathrm{ml})$, non-BA $(0.54 \pm 0.85 \mathrm{ng} / \mathrm{ml})$ and control $(0.24-0.2 \mathrm{ng} / \mathrm{ml})$ groups. The levels of serum IL-13R $\alpha 2$ increase with the severity of the degree of fibrosis. IL-13R $\alpha 2$ at a cutoff level $>0.782 \mathrm{ng} / \mathrm{ml}$ could predict late fibrosis with accuracy of $77.55 \%(p<0.0001)$. IL-13R $\alpha 2$ could differentiate between preserved and disturbed liver architecture at a cut off value of more than $0.42 \mathrm{ng} / \mathrm{ml}$ with an accuracy of $81.6 \%$.

Conclusions: Serum IL-13R 2 not a diagnostic marker for BA however it could be used as a noninvasive marker for detection of advanced liver fibrosis and presence of disturbed liver architecture that helps in patient selection for undergoing Kasai operation. Serum IL-13R $\alpha 2$ could be a future therapeutic target for management of BA patients and any fibrotic liver disease.
\end{abstract}

Key words: cholestasis, liver fibrosis, biliary atresia, interleukin-13R $\alpha 2$, Kasai portoenterostomy.

Address for correspondence

Dr. Haidy M. Zakaria, Quesna Central Hospital, 20048 Quesna, Menoufia, Egypt, phone: +201005768306 , e-mail: drhaydi2000@gmail.com

\section{Introduction}

Biliary atresia (BA) is a neonatal liver disease rapidly progressing to biliary cirrhosis and death in the first years of life, if left untreated [1]. Understanding the various control mechanisms that suppress or inhibit chronic inflammatory reactions is of utmost importance [2].

Interleukin (IL)-4 and IL-13 are related cytokines which induce both pro- and anti-inflammatory effects depending on the cell type they act upon and the nature of the receptors expressed [3]. An even more critical role for IL-13, revealing it as the key mediator of the fibrotic response, has been suggested [4].

IL-13 signals to cells by binding to a complex receptor system composed of IL-4R and two IL-13 binding proteins, IL-13Ra1 and IL-13Ra2. IL-13Ra2 is a $42-\mathrm{kDa}$ monomeric high affinity IL-13 receptor distinct from the more ubiquitously expressed IL-13R $\alpha 1 / \mathrm{IL}-4 \mathrm{R} \alpha$ receptor complex $[5,6]$. It is thought to act primarily as a decoy receptor, sequestering IL-13 from the IL-13Ra1/ IL-4R $\alpha$ complex and thus inhibiting its function $[4,7]$. 
There is evidence that IL-13R 2 may contribute to IL-13-induced TGF $\beta 1$-dependent fibrosis [6].

Increased serum levels of IL-13Ra2 have been detected in both humans and mice in other $\mathrm{TH}_{2}$-dominant immune responses $[8,9]$. However, in humans, its functions, expression regulation with the physiological consequences and its role in IL-4/IL-13 transduction are poorly understood and debatable and remain to be determined $[4,10]$. We aimed to investigate the serum IL-13Ra2 in infants with BA and other neonatal cholestasis, in relation to liver fibrosis, and its utility as a non-invasive marker for selection of BA patients indicated for Kasai portoenterostomy.

\section{Material and methods}

\section{Study population}

This prospective study included 60 infants with neonatal cholestasis in whom liver biopsy was indicated for etiological diagnosis. They were divided into three groups: group 1 - BA group $(n=20)$ all of whom underwent Kasai hepatoportoenterostomy and were followed up for three months post-operatively; group 2 - neglected BA group $(n=20)$ comprising BA patients with a delayed diagnosis (parental reluctance for referrals, inadequate follow-up of neonatal jaundice and misdiagnosis) who lost the chance for corrective surgery; and group 3 - non-BA cholestasis group $(n=20)$ with cholestasis due to causes other than BA. Group 4 comprised 20 apparently healthy infants, age and sex matched, enrolled as a control group. The BA group was further divided 3 months postoperatively according to total bilirubin level into successful outcome (total bilirubin $<2 \mathrm{mg} / \mathrm{dl}$ ) and failed outcome (total bilirubin $\geq 2 \mathrm{mg} / \mathrm{dl}$ ) [11]. All patients were recruited from the outpatient and inpatient clinics of the Pediatric Hepatology Department, National Liver Institute, Menoufia University, Egypt. A fourth group with healthy neonates $(n=20)$ was enrolled in the study as controls. Those infants were attending for routine checkup. Written informed consent was obtained from the parents or the legal guardians of the patients and controls before enrollment in the study. The study protocol conforms to the ethical guidelines of the 1975 Declaration of Helsinki as reflected in a prior approval by the institution's human research committee. The study was approved by the Research Ethics Committee of the National Liver Institute, Menoufia University, Egypt on 3/5/2015.

\section{Etiological diagnosis}

After full history taking, thorough clinical examination, routine investigations, and histopathological as- sessment, the patients were allocated as BA and non-BA. Diagnosis of BA was confirmed by operative cholangiography and/or laparotomy prior to surgery. Routine investigations included total and direct bilirubin, total proteins, albumin, alanine transaminase, aspartate transaminase, alkaline phosphatase, gamma glutamyl transpeptidase, prothrombin time, complete blood count, viral antibodies (immunoglobulin [Ig] M and IgG for rubella, cytomegalovirus, herpes simplex virus type 1 and 2 and hepatitis B virus core), toxoplasma antibodies (both IgM and $\mathrm{IgG}$ ), hepatitis B surface antigen, ultrasonography (US) and Doppler US, with a set of specific laboratory tests according to the expected etiology.

\section{Liver biopsy}

Ultrasonography-guided liver biopsy was conducted for all patients using a Tru-Cut needle (GTA, Quistello, MN, Italy). A core of liver tissue containing at least 5 portal tracts was considered sufficient. Biopsy specimens were fixed in formalin and embedded in paraffin. Five$\mu \mathrm{m}$ thick sections were cut, mounted on a glass slide and stained with hematoxylin and eosin to evaluate pathological changes, with Mason-Trichrome that stains collagen fibers to assess fibrosis, and with Perl's Prussian blue stain which reveals iron deposits. Portal fibrosis and inflammatory activity were assessed using semi-quantitative histopathological scores as described by Russo et al. [11].

\section{Serum IL-13R $\alpha 2$}

Serum samples were collected from all patients and controls in addition to follow-up samples that were collected from BA patients $(n=20)$ three months after the Kasai operation. Samples were stored in aliquots at $-80^{\circ} \mathrm{C}$ until the time of the assay. Quantitative assessment of human serum IL-13Ra2 levels was performed by enzyme linked immunosorbent assay (ELISA). The RayBio Human IL-13Ra2 ELISA kit is an in vitro enzyme-linked immunosorbent assay for the quantitative measurement of human IL-13R 22 in serum and plasma, according to the manufacturer's instructions. The minimum detectable dose of IL-13R 2 is typically less than $0.4 \mathrm{ng} / \mathrm{ml}$.

\section{Statistical analysis}

Descriptive results were expressed as mean \pm standard deviation (SD) or number and percentage. For quantitative data, significance was tested either by the independent sample $t$-test or Mann-Whitney $U$-test according to the nature of the data. A paired $t$-test was used to assess the difference in serum cytokine levels before and after 
the Kasai operation. For qualitative and categorical data significance was tested by the $\chi^{2}$ test or Fisher's exact test. A correlation was tested by Spearman's test. The diagnostic value of serum IL-13R a was assessed by calculating the area under the receiver-operating characteristic (ROC) curves. The cutoff value for optimal clinical performance was determined from the ROC curves. The diagnostic performance was measured as sensitivity, positive predictive value (PPV) and negative predictive value (NPV) and expressed as percentages. Results were considered significant if $p<0.05$. Statistical analysis was performed using SPSS software version 13 (SPSS Inc., Chicago, IL, USA).

\section{Results}

\section{Study population's characteristics}

The current study included 60 infants divided into the BA group $(n=20)$ with the age range $45-90$ days, the neglected BA group $(n=20)$ with the age range 120 210 days, the non-BA group $(n=20)$ with the age range 60-120 days, and the control group with the age range $45-120$ days. All groups were sex matched $(p=0.977)$.

Serum levels of gamma glutamyl transpeptidase (GGT) were significantly higher in the BA group than the non-BA group ( $p=0.0001)$ and in the delayed BA group than early BA and non BA $(p=0.0001)$ groups. The early BA group had significantly lower serum levels of GGT, lower serum alkaline phosphatase (ALP), shorter prothrombin time (PT) and a higher platelet (PLT) count than the neglected BA group $(p<0.0001, p<0.002, p<0.038$, $p<0.007$ respectively). Also, BA patients had significantly higher serum GGT, higher serum ALP and lower PLT than the non-BA group ( $p<0.0001,0.002,0.007$ respectively). The neglected BA group had significantly higher serum levels of GGT, ALP, and lower PLT than the non-BA group $(p<0.0001, p<0.0001, p<0.05$ respectively) (Table 1$)$.

Table 1. Laboratory characteristics of the studied patients

\begin{tabular}{|c|c|c|c|c|c|c|}
\hline & $\begin{array}{c}\text { Naïve BA, } \\
n=20\end{array}$ & $\begin{array}{l}\text { Delayed diagnosed BA, } \\
\qquad n=20\end{array}$ & $\begin{array}{c}\text { Non-BA cholestasis, } \\
n=20\end{array}$ & $\mathrm{P} 1$ & $\mathrm{P} 2$ & P3 \\
\hline \multicolumn{7}{|c|}{ Total bilirubin (mg/dl) } \\
\hline Range & $6.18-24.80$ & $5.60-25.00$ & $4.40-21.00$ & & & \\
\hline Mean \pm SD & $13.30 \pm 4.65$ & $12.71 \pm 4.55$ & $13.36 \pm 8.71$ & 0.617 & 0.543 & 0.665 \\
\hline \multicolumn{7}{|c|}{ Total protein (g/dl) } \\
\hline Range & $4.10-6.30$ & $4.10-6.70$ & $4.10-6.30$ & & & \\
\hline Mean \pm SD & $5.21 \pm 0.61$ & $5.48 \pm 0.78$ & $5.13 \pm 0.61$ & 0.144 & 0.673 & 0.261 \\
\hline \multicolumn{7}{|l|}{ Albumin (g/dl) } \\
\hline Range & $3.20-4.8$ & $3.10-4.30$ & $3.50-4.50$ & & & \\
\hline Mean $\pm S D$ & $3.83 \pm 0.542$ & $3.28 \pm 0.61$ & $3.41 \pm 0.50$ & 0.15 & 0.114 & 0.15 \\
\hline \multicolumn{7}{|c|}{ Prothrombin time (seconds) } \\
\hline Range & $10.80-19.60$ & $11.00-18.90$ & $10.30-34.00$ & & & \\
\hline Mean \pm SD & $13.06 \pm 2.62$ & $14.27 \pm 2.30$ & $14.24 \pm 6.05$ & $0.048 *$ & 0.616 & $0.038 *$ \\
\hline \multicolumn{7}{|l|}{$\mathrm{Hb}(\mathrm{g} / \mathrm{dl})$} \\
\hline Range & $7.30-12.00$ & $8.20-11.30$ & $5.60-13.00$ & 0.655 & 0.159 & 0.167 \\
\hline Mean $\pm S D$ & $9.11 \pm 1.37$ & $9.60 \pm 0.74$ & $9.82 \pm 1.96$ & & & \\
\hline \multicolumn{7}{|c|}{ WBCs $\times\left(10^{3} / \mu \mathrm{l}\right)$} \\
\hline Range & $3.60-18.80$ & $8.40-23.00$ & $3.60-24.00$ & 0.607 & 0.194 & 0.147 \\
\hline Mean \pm SD & $10.8 \pm 4.9$ & $14.34 \pm 4.64$ & $13.27 \pm 5.15$ & & & \\
\hline \multicolumn{7}{|l|}{ PLT $\times\left(10^{3} / \mu \mathrm{l}\right)$} \\
\hline Range & 144-744 & $222-500$ & $163-694$ & 0.213 & $0.05 *$ & $0.007^{*}$ \\
\hline Mean $\pm S D$ & $482 \pm 211$ & $286 \pm 115$ & $360 \pm 164$ & & & \\
\hline \multicolumn{7}{|c|}{ IL-13R $\alpha 2(\mathrm{ng} / \mathrm{ml})$} \\
\hline Range & $0.07-1.81$ & $0.24-29.8$ & $0.08-4.04$ & $<0.0001$ & 0.273 & $<0.0001$ \\
\hline Mean \pm SD & $0.34 \pm 0.37$ & $11.05 \pm 10.9$ & $0.56 \pm 0.18$ & & & \\
\hline
\end{tabular}

P1 - significance between naïve BA and delayed diagnosed BA group, P2 - significance between naïve BA and cholestasis group, P3 - significance between delayed diagnosed and cholestasis group 


\section{Histopathological findings}

The occurrence of higher grades of portal fibrosis were significantly higher in the neglected BA group $(58.8 \%$ had focal porto-portal bridging, $41.2 \%$ had marked bridging). $85 \%$ of BA patients had no fibrosis and $15 \%$ had fibrosis. The entire non-BA group (100\%) did not reach porto-portal bridging (mild fibrous expansion), $p<0.0001$. Patients with neglected BA had statistically significantly higher disturbed liver architecture, ductular proliferation and bile plugs $(p<0.0001, p<0.0001, p=0.008$, respectively). The number of giant cells was significantly higher in the non-BA group than other groups $(p=0.036)$.

\section{Comparison of preoperative serum IL-13R $\alpha 2$ in the studied groups}

Serum levels of IL-13R 2 were significantly higher in the neglected BA group $(11.05 \pm 10.9 \mathrm{ng} / \mathrm{ml})$ than in the early BA $(0.34 \pm 0.37 \mathrm{ng} / \mathrm{ml})$, non-BA $(0.54 \pm 0.85 \mathrm{ng} /$ $\mathrm{ml})$ and control group $(0.24-0.2 \mathrm{ng} / \mathrm{ml})(p<0.0001$, $p<0.0001, p<0.0001$ respectively). Levels were higher in both BA and non-BA groups than the control group $(p<0.0001)$, while no significant difference between BA and the non-BA groups was detected $(p=0.273)$.

\section{Clinical performance of preoperative serum IL-13R $\alpha 2$ in predicting fibrosis in BA patients and non-BA patients}

The levels of serum IL-13Ra2 increase with the severity of fibrosis. IL-13R 2 at a cutoff level $>0.782 \mathrm{ng} /$

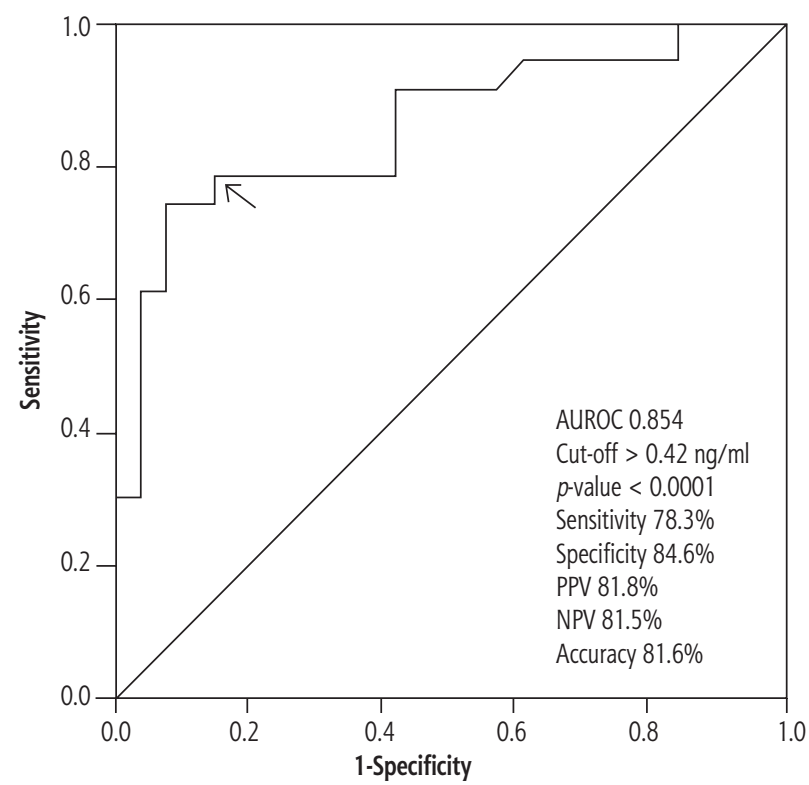

Fig. 1. IL-13R 2 could differentiate between preserved and disturbed liver architecture at a cut-off value of more than 0.42 with $84.6 \%$ specificity, $78.3 \%$ sensitivity, PPV $81.8 \%$, NPV $81.5 \%$, and an accuracy of $81.6 \%$ with higher levels in the latter $\mathrm{ml}$ could predict late fibrosis with $92.3 \%$ sensitivity, 60.9\% specificity, 72.7\% PPV, 87.5\% NPV and accuracy of $77.55 \%(p<0.0001)$. IL-13R 22 could differentiate between preserved and disturbed liver architecture at a cut-off value of more than 0.42 with $84.6 \%$ specificity, $78.3 \%$ sensitivity, PPV $81.8 \%$, NPV $81.5 \%$, and an accuracy of $81.6 \%$, with higher levels in the latter (Fig. 1).

\section{Correlation of IL-13R $\alpha 2$ with the studied parameters in all individuals}

Serum levels of IL-13Ra2 were statistically significantly correlated with age, white blood cells (WBCs), and ultrasound spleen length $(p<0.0001, p=0.006$, $p=0.009$ respectively). On the other hand there was no significant correlation as regards other parameters $(p>0.05)$.

\section{Discussion}

Type 2 cytokine responses, described as anti-inflammatory mediators, are critically involved in tissue repair. Others clearly demonstrated many of them as proinflammatory, with unidentified mechanisms that regulate beneficial regeneration versus pathological fibrosis [12]. A more critical role for IL-13 had been suggested as a key mediator of the fibrotic response in many chronic infectious diseases through the induction of a receptor formerly considered to function only as a decoy receptor [13-15].

As far as we know, this is the first study on serum IL-13Ra2 in BA in humans. In the current study, serum IL-13Ra2 was detected in all the studied groups, with the highest levels among the delayed diagnosed BA. It was found that the cell surface IL-13R 22 can be induced in response to high concentrations of IL-4 or IL-13 and regulated by tumor necrosis factor [6]. Evidence suggests the existence of an intracellular pool of receptors capable of rapidly populating the cell surface in response to inducing agents [16]. Enzymatic cleavage from the cell surface may increase the soluble form of IL-13Ra2, thus increasing its level [17]. The high level of IL-13Ra 2 could be attributed to marked elevation in its gene and protein expression as it had been reported after infection by Schistosoma mansoni in both humans and mice [18]. Consistently with our results, Hussein et al. reported the serum level of IL-13Ra2 in atopic asthma, allergic rhinitis, and atopic dermatitis respectively [19]. The impact of the disease itself and age group may explain the higher level in neglected BA than the results Hussein et al.

The control group had a minimal level of IL-13Ra2. In agreement with us, Hussein et al. (2011) Khodoun et al. and Chen et al. (2009) did not detect signifi- 
cant levels of IL-13R $\alpha 2$ in the control groups [19-21]. However, the control group had a significantly lower level than the newly diagnosed BA and the cholestatic groups, which may be attributed to the presence of different grades of liver fibrosis in newly diagnosed BA and the cholestatic groups. Figueiredo et al. suggested that an increase in IL-13 early in the fibrogenesis process saturates the receptor and makes IL-13 freely available in the microenvironment [22].

Assessing IL-13 production and its receptors' association in different degrees of liver fibrosis remains to be answered. On the other hand, serum IL-13Ra2 level could not differentiate between early diagnosed $\mathrm{BA}$ and non-BA as a cause of cholestasis. This comparable level of IL-13R $a 2$ may indicate that it is not disease specific.

A significant positive correlation between the level of IL-13R 22 and age was detected in this study. Prolonged exposure to proinflammatory and Th2 cytokines (TNF- $\alpha$ and IL-13) in prolonged inflammation may be the main factor. Leukocytosis was positively correlated with serum level of IL-13Ra2. IL-13 upregulates cysteinyl leukotrienes (cysLTs), which in turn increases the recruitment of leukocytes and their biosynthesis of cysLTs [23].

IL-13Ra2 expression is most closely correlated, but not limited to, expression of mesenchymal signature genes [24, 25]. IL-13 directly induces expression of collagen I and other critical fibrosis-associated genes, e.g., $\alpha$-smooth muscle actin and connective tissue growth factor, in hepatic stellate cells. IL-13 is known as a potent inducer of matrix metalloproteinases- 9 and cathepsin-based proteolytic pathways which stimulate cleavage of latency-associated peptide and thus activate TGF- $\beta$ [9]. Also, direct cleavage by MMP-8 contributes to the solubilization of IL-13Ra2 and increases its level. Others found that prevention of IL-13Ra2 expression reduced production of TGF- $\beta_{1}$ and marked downregulation of collagen, and regression of fibrosis [6].

In this study, the levels of serum IL-13Ra2 increased with the severity of fibrosis. Higher grades of fibrosis in neglected BA could be an additional factor explaining the highest IL-13R 2 levels. IL-13Ra2 had a very good accuracy in differentiating patients with preserved and those with disturbed liver architecture, with higher levels in the latter. Thus we can select patients indicated for the Kasai operation.

\section{Conclusions}

Serum IL-13Ra2 not a diagnostic marker for BA, but it could be used as a noninvasive marker for de- tection of advanced liver fibrosis and presence of disturbed liver architecture, which helps in patient selection for undergoing the Kasai operation. Serum IL-13Ra 2 could be a future therapeutic target for management of BA patients and any fibrotic liver disease.

\section{Acknowledgements}

We would like to express our gratitude to our colleagues in the Department of Pediatric Hepatology, Gastroenterology and Nutrition, National Liver Institute, Menoufia University, who provided insight and expertise that greatly assisted the research. We would also like to thank all the patients who participated in the study.

\section{Disclosure}

Authors report no conflict of interest.

\section{References}

1. Sokol RJ, Shepherd RW, Superina R, et al. Screening and outcomes in biliary atresia: summary of a National Institutes of Health workshop. Hepatology 2007; 46: 566-581.

2. Hoffmann KF, McCarty TC, Segal DH, et al. Disease fingerprinting with cDNA microarrays reveals distinct gene expression profiles in lethal type 1 and type 2 cytokine-mediated inflammatory reactions. FASEB J 2001; 15: 2545-2547.

3. Li J, Bessho K, Shivakumar P, et al. Th2 signals induce epithelial injury in mice and are compatible with the biliary atresia phenotype. J Clin Invest 2011; 121: 4244-4256.

4. Chiaramonte MG, Mentink-Kane M, Jacobson BA, et al. Regulation and function of the interleukin 13 receptor a 2 during a T helper cell type 2-dominant immune response. J Exp Med 2003; 197: 687-701.

5. Lupardus PJ, Birnbaum ME, Garcia KC. Molecular basis for shared cytokine recognition revealed in the structure of an unusually high affinity complex between IL-13 and IL-13Ra2. Structure 2010; 18: 332-342.

6. Fichtner-Feigl S, Strober W, Kawakami K, et al. IL-13 signaling through the IL-13alpha 2 receptor is involved in induction of TGF-beta 1 production and fibrosis. Nat Med 2006; 12: 99-106.

7. Wood N, Whitters MJ, Jacobson BA, et al. Enhanced interleukin (IL)-13 responses in mice lacking IL-13 receptor $\alpha 2$. J Exp Med 2003; 197: 703-709.

8. Lumsden RV, Worrell JC, Boylan D, et al. Modulation of pulmonary fibrosis by IL-13Ra2. Am J Physiol Lung Cell Mol Physiol 2015; 308: 710-718.

9. Mentink-Kane MM, Cheever AW, Thompson RW, et al. IL-13 receptor alpha 2 down-modulates granulomatous inflammation and prolongs host survival in schistosomiasis. Proc Natl Acad Sci U S A 2004; 101: 586-590.

10. Fichtner-Feigl S, Terabe M, Kitani A, et al. Restoration of tumor immunosurveillance via targeting of interleukin-13 receptor- $\alpha 2$. Cancer Res 2008; 3467: 68-75.

11. Russo P, Magee JC, Boitnott J, et al. Design and validation of the biliary atresia research consortium histologic assessment system for cholestasis in infancy. Clin Gastroenterol Hepatol 2011; 9: 357-362.e2. 
12. Opal SM, DePalo VA. Anti-inflammatory cytokines. Chest 2000; 117: 1162-1172.

13. Pandya H, Gibo DM, Garg S, et al. An interleukin 13 receptor a 2-specific peptide homes to human Glioblastoma multiforme xenografts. Neuro Oncol 2011; 14: 6-18.

14. Daines MO, Hershey GKK. A novel mechanism by which interferon- $\gamma$ can regulate interleukin (IL)-13 responses. Evidence for intracellular stores of IL-13 receptor $\alpha-2$ and their RAPID mobilization by interferon-gamma. J Biol Chem 2002; 277: 10387-10393.

15. Prikk K, Maisi P, Pirilä E, et al. Airway obstruction correlates with collagenase-2 (MMP-8) expression and activation in bronchial asthma. Lab Invest 2002; 82: 1535-1545.

16. Wang W, Shen YX, Li J, et al. Enhanced expression of the decoy receptor IL-13Ralpha2 in macrophages of Schistosoma japonicum-infected mice. Chin Med J (Engl) 2009; 122: 1650-1654.

17. Hussein $Y$, Ahmad A, Ibrahem M, et al. Interleukin 13 receptors as biochemical markers in atopic patients. J Invest Allergol Clin Immunol 2010; 21: 101-107.

18. Khodoun M, Lewis C, Yang J-Q, et al. Differences in expression, affinity, and function of soluble(s) IL-4Ra and sIL-13Ra2 suggest opposite effects on allergic responses. J Immunol 2007; 179: 6429-6438.

19. Chen W, Sivaprasad U, Tabata Y, et al. IL-13R alpha 2 membrane and soluble isoforms differ in humans and mice. J Immunol 2009; 183: 7870-7876.

20. Figueiredo AL, Domingues AL, Melo WG, et al. Receptor Antagonist of IL-13 Exerts a Potential Negative Regulation During Early Infection of Human Schistosomiasis. Scand J Immunol 2016; 84: 284-290.

21. Provost V, Langlois A, Chouinard F, et al. Leukotriene D4 and interleukin- 13 cooperate to increase the release of eotaxin- 3 by airway epithelial cells. PLoS One 2012; 7: e43544.

22. Verhaak RG, Hoadley KA, Purdom E, et al. Integrated genomic analysis identifies clinically relevant subtypes of glioblastoma characterized by abnormalities in PDGFRA, IDH1, EGFR, and NF1. Cancer Cell 2010; 17: 98-110.

23. Phillips HS, Kharbanda S, Chen R, et al. Molecular subclasses of high-grade glioma predict prognosis, delineate a pattern of disease progression, and resemble stages in neurogenesis. Cancer Cell 2006; 9: 157-173.

24. Mata-Santos HA, Dutra FF, Rocha CC, et al. Silymarin reduces profibrogenic cytokines and reverses hepatic fibrosis in chronic murine schistosomiasis. Antimicrob Agents Chemother 2014; 58: 2076-2083.

25. Chen W, Tabata Y, Gibson AM, et al. Matrix metalloproteinase 8 contributes to solubilization of IL-13 receptor a 2 in vivo. J Allergy Clin Immunol 2008; 122: 625-632. 